because muscle fibers were relatively slender. At new born infant end plates connected to long nerve fiber increased remarkably.

\title{
2. A Study on Degenerative Changes of Neuromuscular Junction in Skeletal Muscle after Denervation by Electron Microscope
}

\author{
Yoshito KIRITA, Takemichi OHASHI \\ Orthopedic Surgery, Kyoto University Medical School \\ (Director: Prof. E. Kondo)
}

Using albino mice, normal figures and degenerative changes of neuromuscular junction in a gastrocunemius after denervation of sciatic nerve by electron microscope are examined.

In control animals, a few number of axon terminal branches are in contact with ditches of sarcoplasm surface with intervening crevice, "Primary Synaptic Cleft", formed by both axolemma and sarcolemma. The sarcolemma has many crease-like crevices, "Second Synaptic Cleft", directly connected with the "Primary Synaptic Cleft".

Both endplate sarcoplasm and terminal axoplasm contain various organellae such as mitochondria, endoplasmic reticulum, synaptic vesicle and so on.

On the opposite side of neuromuscular junction, the terminal axon is covered by Schwann cell.

(1) 24 hours after denervation:

The mitochondriae are swollen and their cristae are obscure. The vesicles decrease markedly.

(2) 48 hours after denervation:

The axon becomes atrophied and the primary synaptic cleft is enlarged. Moreover, organellae of the axon decrease in number.

(3) 3-5 days after denervation:

Almost all of terminal axons disappear and Schwann cells protrude into the synaptic region. These Schwann cells are hypertrophied and contain abundant organellae.

(4) 7 days after denervation:

The Schwann cells replace completely the dead spaces which were made by disappearance of terminal axons, and in connection with sarcolemma with an internenning cleft. 